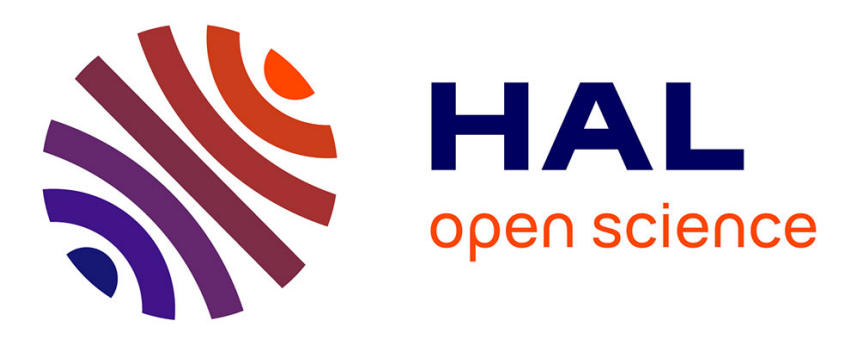

\title{
On the relationship between palate shape and articulatory behavior
}

Jana Brunner, Susanne Fuchs, Pascal Perrier

\section{To cite this version:}

Jana Brunner, Susanne Fuchs, Pascal Perrier. On the relationship between palate shape and articulatory behavior. Journal of the Acoustical Society of America, 2009, 125 (6), pp.3936-3949. 10.1121/1.3125313 . hal-00391836

\section{HAL Id: hal-00391836 https://hal.science/hal-00391836}

Submitted on 5 Jun 2009

HAL is a multi-disciplinary open access archive for the deposit and dissemination of scientific research documents, whether they are published or not. The documents may come from teaching and research institutions in France or abroad, or from public or private research centers.
L'archive ouverte pluridisciplinaire HAL, est destinée au dépôt et à la diffusion de documents scientifiques de niveau recherche, publiés ou non, émanant des établissements d'enseignement et de recherche français ou étrangers, des laboratoires publics ou privés. 
On the relationship between palate shape and articulatory behavior

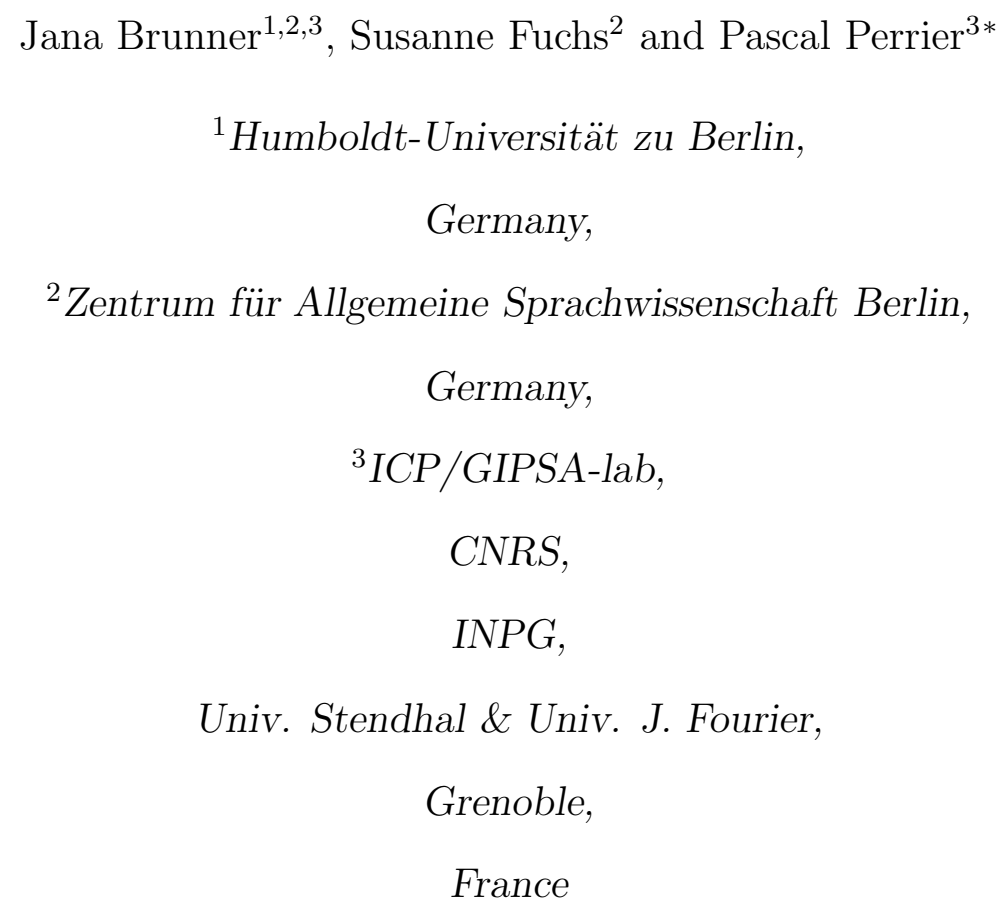

(Dated: March 26, 2009) 


\begin{abstract}
In this study the acoustic and articulatory variability of speakers with different palate shapes were compared. Since the cross-sectional area of the vocal tract changes less for a slight change in tongue position if the palate is domeshaped than if it is flat, the acoustic variability should be greater for flat palates than for domeshaped ones. Consequently, it can be hypothesized that speakers with flat palates should reduce their articulatory variability in order to keep the acoustic output constant. This hypothesis was tested on 32 speakers recorded via EPG and acoustics. The articulatory and acoustic variability of some of their vowels and $/ \mathrm{j} /$ was measured. Indeed, the results show that the speakers with flat palates reduce their variability in tongue height. There is no such trend in acoustic variability.
\end{abstract}

PACS numbers: 43.70.-h, 43.71.Bk, 43.70Mn 


\section{INTRODUCTION}

Since Stevens' seminal paper (Stevens (1972)) it is known that the relationship between articulation and acoustics is nonlinear. In the present study we make use of this nonlinearity in order to investigate speakers' control of variability. Basically, we compare speakers for whom theoretical models of articulatory-acoustic relations predict that they can allow for much articulatory variability without having as much variability in the acoustic output with speakers for whom the models suggest that they cannot allow for so much articulatory variability, because then the acoustic output would be too variable. The differences in speakers' variability are assumed to exist because of differences in morphology.

Let us consider two ideal and very different palate shapes in the coronal plane, the one very flat and the other very curved or domeshaped (cf. Figure 1). Let us also consider for the sake of simplicity and clarity in the demonstration, that both palates would have the same distance $a$ between the molars (symbolized as squares). The speaker with the domeshaped palate (right side in the figure) then has to move his or her tongue further up in order to have the same cross-sectional area $A$ as the speaker with the flat palate. The width of the vocal tract at the height of the tongue surface is then $a_{t}$, which is smaller than $a$. For the flat palate $a_{t}$ would be equal to $a$ and is therefore not given in the figure.

\section{FIGURE 1}

If the tongue is now raised by $\Delta d$, the distance between tongue and palate changes to $d_{c}-\Delta d$ for the domeshaped palate (c stands for curved) and $d_{f}-\Delta d$ for the flat palate. The difference between the original and the new area is for the flat palate

$$
\Delta A_{f}=a * \Delta d
$$

For the domeshaped palate, if we approximate the palate sides with straight lines,

$$
a^{\prime}=\frac{a d_{c}}{h_{c}}
$$

*Jana Brunner brunner@zas.gwz-berlin.de 
the difference in the cross-sectional area is

$$
\Delta A_{c}=a \Delta d \frac{d_{c}}{h_{c}}
$$

Given that $h_{c}$ (height of the domeshaped palate) is greater than $d_{c}$ (distance between tongue and domeshaped palate) the fraction $\frac{d_{c}}{h_{c}}$ is smaller than 1. Consequently, a comparison between equations 1 and 3 shows that $\Delta A_{c}$ is smaller than $\Delta A_{f}$. This means that for the same change in articulation the area changes more for the flat palate than for the domeshaped one. Hence, for the same tongue movement, one of the perceptually relevant characteristics of the vocal tract (i.e. the constriction area) will change to a larger extent if the palate is flat than if it is domeshaped.

Under the assumption that speakers should be interested in keeping the acoustic output constant, it is hypothesized that speakers should compensate for these differences in the acoustics caused by differences in palate shape: Speakers with flat palates should reduce their articulatory variability in order to keep the acoustic variability within an acceptable range.

In fact, evidence for differences in articulatory variability associated with these kinds of vocal tract differences has previously been found. Perkell (1997) compared six speakers with different palatal vaults who produced $/ \mathrm{i} /, / \mathrm{I} /$, and $/ \varepsilon /$. He found that the speaker with the shallowest vault showed the smallest variability in tongue height for the three vowels. The result has been supported by Mooshammer et al. (2004) for the more crowded vowel inventory of German. They compared three speakers, two of them with a domeshaped palate and one with a flat palate, and found that the speaker with a flat palate had a lower articulatory variability as compared to the other speakers.

In this context, the study deals with the following questions: (1) Is the acoustic variability of speakers comparable, no matter what their palate shape is? (2) Is the articulatory variability greater for speakers with domeshaped palates than for speakers with flat palates?

Whereas question (2) deals with the relation between articulatory variability and the palate shape, question (1) deals with the relation between acoustic variability and the palate 
shape.

In order to investigate these questions thirty-two speakers were recorded acoustically and via Electropalatography. The formant variability and the variability of the linguo-palatal contacts was calculated. Furthermore, the shape of the speakers' palates was estimated. If speakers with flat palates turn out to have less articulatory variability but the same acoustic variability as other speakers this would support the hypothesis that speakers with a flat palate reduce their articulatory variability in order to keep the acoustic variability at a level which the listener can tolerate.

The following section describes the EPG experiment. Section III describes its results. In section IV the results are discussed.

\section{METHODS}

The first part of this section (section II.A) gives information about the speakers, their gender and which language they speak. Section II.B describes the variation in palate shape in humans and how we determined the palate shape of our speakers. Section II.C describes the recording procedure. Section II.D deals with the problem of different crowdedness of the phoneme inventory in the different languages of our speakers, which could influence our measurements of variability. Section II.E describes our measurements of articulatory variability. Articulatory variability was assessed in three ways: (1) as the coefficient of variation of the percent of contact over the complete segment, (2) as the standard deviation of the center of gravity at the articulatory target and (3) as the coefficient of variation of the number of contacts in a row. Finally, in section II.F we describe how we assessed acoustic variability, i.e. as the standard deviation of the first three formants over the complete segment. Section II.G describes the statistics carried out. 


\section{A. Speakers}

Since the question investigated here is not bound to a certain language but to human speech production in general, 32 speakers of languages featuring different phonological characteristics were recorded:

- two speakers of Bulgarian (one male, one female)

- three speakers of Polish (one male, two females)

- eleven speakers of English (five English (two males, three females), four Scottish (three males, one female), one American (male), and one Australian (male))

- ten speakers of German (six males, four females)

- six speakers of Norwegian (Urban East Norwegian, two males, four females).

We thus had 15 female and 17 male speakers. Since the gender can be expected to influence at least the acoustic variability we carried out statistical tests for a gender effect in our data.

\section{B. Characterisation of the palate shape}

Human palates differ considerably both in size and curvature. In Vorperian et al. (2005)'s sample of adult palates length variation goes from about 3.7 to $5.2 \mathrm{~cm}$. However, the human palate changes from birth to adulthood. The greatest changes in palate shape take place in very early childhood. Vorperian et al. (2005) investigated the growth and restructuring of the vocal tract until the age of six. They found that the hard palate grows very rapidly until the age of 18 months, where it has reached $80 \%$ of its adult mature size. Cheng et al. (2007) show that there are significant changes in palate shape until the age of 11 which go together with differences in articulatory control (e.g. place of articulation, amount of palatal contact). After age 11 the shape of the palate stays about the same even if the articulatory 
control develops further. Hiki and Itoh (1986) found that the adult palate is typically deeper than the child's palate.

Fitch and Giedd (1999) showed that from puberty onwards there is a gender difference in vocal tract anatomy. These differences are most pronounced in the lips and the pharynx (see also Fant (1966)) which are disproportinately larger in men than in women, and this difference goes beyond the one explainable by body size. Fitch and Giedd (1999), however, did not find changes with regard to palate size between males and females except for those which are due to differences in body size.

Cheng et al. (2007) found possible gender differences in palatal contact, which, however, were inconsistent. The authors conclude that "no genuine differences may exist between the sexes." (p.387).

Since our speakers were all at least 25 years old, one can assume that they have the typical more curved adult palate and adult articulatory patterns. Even if clear gender differences have so far not been found in the literature, we will carry out statistical tests in order to see whether there are gender differences in palatal shape and variability.

In this study we focus on palatal doming. In order to investigate the relationship between palatal doming and variability one needs to determine the curvature of the palate. Measurements were made from the cast of each palate which existed in the form of an EPG palate.

At first the coordinates of each of the 62 electrodes were measured using a caliper. In order to do so the EPG palate was placed on a photocopier and a high quality copy was made on which two dimensions of the placement of each electrode could be seen. A coordinate system was set up on the photocopy with the point of origin in the leftmost, most posterior electrode. The abscissa was set up from the two outermost electrodes on the most posterior row of electrodes. The ordinate was set up perpendicular to the abscissa. Then the $\mathrm{x}$ and $\mathrm{y}$ values of each electrode were measured with the caliper.

In order to measure the third dimension the artificial palate was put in the dental cast of the subject. A plexiglas disc with a small hole in it was placed on top. The disk was 
moved so that the hole was exactly above the electrode to be measured. The caliper was put through the hole and the distance from the electrode to the plexiglas disc was measured.

A result of this can be seen in Figure 2. We selected the sixth row from the front for calculating the coefficient $\alpha$. This coefficient models the relationship between sagittal distance and area function and gives information about palatal curvature for a constant tongue curvature (cf. appendix A). The sixth row was taken because it presents the middle of the palatal zone. Since the electrode placing of the EPG palate is carried out according to certain anatomical landmarks (Wrench (2007)) the measurement is on the whole comparable for all palates.

FIGURE 2

A parabolic approximation with two coefficients was carried out for the measured points of row 6 . The palatal shape could now be described by

$$
y(x)=a x^{2}+b
$$

and $\alpha$ was calculated as

$$
\alpha=\frac{4}{3 \sqrt{|a|}}
$$

by assuming that the tongue is flat (cf. Perrier et al. (1992) for an explanation). Assuming that the tongue curvature remains constant, a high $\alpha$-value corresponds to a flat palate and a low value to a domeshaped palate.

\section{EPG recordings}

The speakers were recorded via Electropalatography (Reading System). All speakers were experienced in speaking with an artificial palate and had taken part in several EPG studies before. The exeriments started after speakers had become comfortable with the palate and their speech sounded normal to the investigator. The length of this period varied but usually took a couple of minutes. For the Norwegian and all but one of the English subjects the WinEPG was used. The German, Polish, Bulgarian, and one English subject 
were recorded with EPG 3. A parallel acoustic recording was carried out with a DAT recorder for the German, Bulgarian, and Polish subjects and one English subject. For the other subjects the acoustic recording was carried out via WinEPG. The sampling rate of the articulatory data was $100 \mathrm{~Hz}$. During the recording, if an error or dysfluency occurred, additional repetitions were recorded. The speakers produced between 0 and 11 errors per recording, the average error rate was $1.4 \%$.

The sounds to be investigated were the consonant $/ \mathrm{j} /$ and the vowels /i/, and /e/ with their lax counterparts /I/ and / $\varepsilon /$. In some languages the tense-lax contrast goes together with a length contrast. In German, tense vowels are long in stressed position (cf. e.g. Hoole and Mooshammer (2002)) so that the tense vowels in our sample are all long since they occur in stressed position. In Norwegian there is also a tense-lax contrast which is realized by differences in quantity and quality, even if the quality difference is, in contrast to German, predictable from either length or stress (Kristoffersen (2000)). In English there is also a length contrast; however, the quality contrast is, in comparison to the other two languages, more pronounced (e.g. Peterson and Barney (1952), Hillenbrand et al. (1995) for American English).

The sounds in our sample were choosen because the vocal tract is rather narrow during their production and consequently an influence of the palate shape can be expected. In order to make the data in the different languages comparable, nonsense words were used rather than real words. Doing so it was possible to take the same items for all the languages. Since some of the sounds do not have phonemic status in all the recorded languages not all the speakers were recorded speaking all items. For Bulgarian and Polish speakers no lax vowels were recorded; for English there was no /e/ (but $/ \varepsilon /$ ).

The items in which the sounds were embedded were: /'titi/, /'trti/, /'tetə/, /'tetə/ (for the English speakers /'tetər/ or /'tetə/) and /'jaja/. The carrier phrases differed from language to language:

- for Bulgarian: Kazah ... na teb. (I have said ... to you.) 
- for Polish: Powiedzialem ... do ciebie. (I said ... to you.)

- for German: Habe ... gesagt. ((I) have said ...)

- for English: Say ... please.

- for Norwegian: Jeg sa ... 'a vet du. (I said ... you know.)

As one can see, the phonetic contexts for the target words differ across the languages. For Bulgarian the test word is preceded by a $/ \mathrm{x} /$, for Polish by an $/ \mathrm{m} /$, and for the other languages by some kind of centralized vowel. Since carryover effects have been found to spread over two or three segments (Daniloff and Hammarberg, 1973), one could assume that the different preceding sounds could have an influence on the measurements carried out on the target vowel and consonant. However, Daniloff and Hammarberg found these wide spreadings only for slow speech. Since our speakers spoke with normal speed one can assume that the influence is minor. Additionally, we did not analyze the first phone of the test word, but its presence means that the consonant immediately preceding the target vowel was always the same.

Each sentence was repeated 30 times in randomized order. The beginning and end of each segment of interest was labelled in the acoustic signal using PRAAT 4.2.17 (Boersma and Weenink, 1992-2004):

- sonorant onset and offset for the medial sonorant $/ \mathrm{j} /$ as the middle of the formant transitions between the surrounding vowels and the sonorant.

- onset and offset of the second formant for the vowels in stressed position.

\section{Influence of the size of the phoneme inventory}

There are a couple of studies investigating the influence of the size of the phoneme inventory on token-to-token variability (e.g. Tabain and Butcher (1999) for consonants, Dixon (1980) for vowels). The basic question behind these studies is whether speakers reduce 
the variability to a level which allows clear boundaries between the phonemes. Although the results of these studies are not entirely consistent regarding complete phoneme inventories (Tabain and Butcher found that there is no influence for consonants, but Dixon found that there is one for vowels) the possibility that this influence exists has to be taken into account in the present study.

In order to find out whether the languages in our study are comparable with respect to the sounds studied here, the phoneme inventories were compared. However, as suggested by the results of Tabain and Butcher and of Dixon, a more crowded inventory in one domain, e.g. vowels, does not necessarily influence the variability of productions in another domain, e.g. consonants. In order to account for the potential influence of the phoneme inventory on vowel variability, we compared the number of unrounded front vowels in the languages of the study. Rounded front vowels were not counted since we hypothesized that the existence of rounded front vowels in German and Norwegian should not influence the variability of the unrounded front vowels considerably, either articulatorily or acoustically. Indeed, lip rounding and spreading presents an additional degree of freedom and is very likely to provide a clear distinction between the spaces of the articulatory representations of these two vowel categories. Acoustically, lip rounding generates a strong frequency decrease of the lowest front cavity resonance in a way that is very different from the consequence of local tongue displacements. For the approximant $/ \mathrm{j} /$, we counted places of articulation for the respective manner of articulation for lingual consonants. The information about the phoneme inventories of Bulgarian and German was taken from The Handbook of the IPA (IPA, 1999). Our sources for the other languages were Kristoffersen (2000) for Norwegian; Gimson and Cruttenden (2001) for British English, and Jassem (2003) for Polish.

\section{TABLE I}

All five languages have just one palatal or velar approximant (cf. table I). With respect to the vowels, English, Norwegian, and German are comparable in terms of the number of places of articulation. Polish and Bulgarian have fewer unrounded front vowels. We therefore tested if there is an influence of the number of unrounded front vowels on articulatory and 
acoustic variability.

\section{E. Measuring articulatory variability}

Articulatory variability was measured in three ways:

1. as the coefficient of variation of the percent of contact over the complete segment

2. as the standard deviation of the center of gravity (a measurement of tongue position variation)

3. as the coefficient of variation of the number of contacts at the articulatory target (a measurement of tongue height variation)

\section{Coefficient of variation of the percent of contact - "POC-variability"}

The percent of contact describes whether there is much or little contact without specifying where the contact occurs. The articulatory variability of this index was calculated as follows:

- Calculation of the percent of contact for each EPG frame within the acoustically measured segment

- Temporal alignment of the 30 repetitions for each speaker and each item

- Calculation of a mean and the standard deviation for each sample of the normalized 30 repetitions

- Calculation of a mean of the standard deviations over all samples

- Normalisation of this value at the mean percent of contact

- Calculation of the maximally observed variability. 
These steps will now be described in detail.

At first, the percent of contact was calculated for each EPG frame within the measured time interval as:

$$
p_{c}=\frac{n_{c} * 100}{62}
$$

with $n_{c}=$ number of contacts and 62 as the maximal number of contacts (Hardcastle and Gibbon, 1997).

Figure 3 illustrates this method. The left subplot shows the EPG frames of a production of /i/ surrounded by /t/ by speaker E1 (English speaker 1, male). In the beginning there is more contact because the preceding consonant is /t/. The closure, however, cannot be seen any more since the figure only shows the frames corresponding to the segment of the acoustic signal that has a clear formant structure. Then there is less and less contact. Towards the end there is again more contact because the phoneme following the /i/ is again / $\mathrm{t} /$.

\section{FIGURE 3}

The right subplot in the Figure shows the POC for each of the frames in the left subplot. As one can see there is a higher percentage of contact in the beginning (when consonantal characteristics are still present), the POC falls in the middle of the segment (during/i/), and towards the end the POC rises again because of the second $/ \mathrm{t} /$. These calculations were carried out for the 30 repetitions of each item.

After this calculation data were split according to speaker and item. As one can imagine, the 30 repetitions of one item usually differed in duration. In order to calculate standard deviations over the complete segment, however, they had to be time-aligned. Therefore, they were aligned nonlinearly according to an algorithm proposed by Lucero and colleagues (Lucero et al., 1997 and Lucero and Koenig, 2000). The algorithm aligns beginning, end, maxima and minima of the POC-signal. The transformation of the time scale is therefore nonlinear.

After this temporal alignment, a mean value and the standard deviation of the 30 repetitions were calculated for each of the sample points of the nonlinearly aligned repetitions. The mean of all the standard deviations (one for each sample point) was calculated separately 
for each item and each speaker (across the 30 repetitions of one item). When the data were split according to the item (and thus according to the target sound), a significant positive correlation between mean and standard deviation was found for $/ \varepsilon /$ and $/ \mathrm{j} /$. Hence, the standard deviations (one for each sample point) of the POC were normalized by the mean value of the POC. We thereby obtained the coefficient of variation. The resulting value was treated as a measure of the articulatory variability of one item uttered by a speaker and will be called POC-variability.

As a final step we calculated the maximally observed variability. This step is grounded in the following reasoning. According to the argumentation in the introduction, one can expect speakers with flat palates to reduce their articulatory variability. This, however, does not mean that speakers with domeshaped palates should always have a high articulatory variability. For all speakers it is possible that they have less variability than they could allow for. The speakers with domeshaped palates, however, have a greater range of possible levels of variation since the articulatory variability they can allow for without changing the acoustic output considerably is higher. ${ }^{1}$. What we are interested in is consequently the maximum possible variability for a certain palate shape. It is therefore important not only to look at the observed variability, but at the maximally observed variability for a certain palate shape. In order to do so, we distributed palate shapes across three groups: flat palates, medium palates and domed palates. The division was carried out linearly: Since the highest observed $\alpha$ was 2.24 and the lowest was 1.23 , the border between domed and medium palates was placed at 1.57 and the one separating medium and flat palates at 1.91.

In order to get the maximum possible variability in each palate shape group, for each segment and each palate group the upper third (rounded to the nearest higher integer) of the observed POC-variability was considered. To give an example for domed palates, for the 10 speakers with a domed palate ( $\alpha$ between 1.23 and 1.57) the following POC-variabilities were observed for /j/: 0.1139, 0.1194, 0.1239, 0.1254, 0.1279, 0.1736, 0.1783, 0.2199, 0.2223, 0.3318. The upper third corresponds to the four speakers with the highest variability values: $0.3318,0.2223,0.2199,0.1783$. These four values are the maximally observed articulatory 
variability for $/ \mathrm{j} /$ for speakers with domed palates. This variability will be called maximally observed POC-variability.

\section{Standard deviation of the center of gravity: "COG-variability"}

In contrast to the first measurement which calculated variability over the complete segment, the second measurement is one which corresponds to one moment in time only and not to a complete segment. The reason for doing this was to test whether we would get the same result for canonical tongue positions.

Briefly, the following calculations were carried out:

- Determination of an articulatory target for each production

- Calculation of the center of gravity for the target EPG frame

- Calculation of the standard deviation of the mean COG for the 30 repetitions of one item uttered by the same speaker

- Calculation of the maximally observed COG-variability

First, in each production we labelled a point in time which could be seen to be the articulatory target position of the sounds. The definition of this articulatory target relies on the assumption that consonants have more linguo-palatal contact than vowels. A transition from a vowel to a consonant will therefore involve an increase of the contact whereas a transition from a consonant to a vowel will involve a reduction of contact. The articulatory target for a vowel was therefore the frame with the least contact within the acoustically measured segment. The articulatory target for a consonant was the frame with the most contact within the acoustically measured segment.

This method is demonstrated by figure 3. The articulatory target of the /i/-production shown there would be the 4 th and 5 th sample in the figure since there is the least contact in this production. 
For the EPG frame at this articulatory target position we calculated the center of gravity, which is a measure of the mean location of contact along the anterior-to-posterior axis of the palate (Hardcastle et al. (1991)). If the COG is low this means that there is more contact in the anterior region; if it is high there is more contact in the posterior region of the palate.

The center of gravity was calculated according to the following formula:

$$
C O G=\frac{R 1+2 R 2+3 R 3+4 R 4+5 R 5+6 R 6+7 R 7+8 R 8}{\sum(\text { contacts })}
$$

where $\mathrm{R} 1$ to $\mathrm{R} 8$ denote the contacts observed in the horizontal rows of the palate from the most anterior to the most posterior position.

Since there was no correlation between the standard deviation of this coefficient and the mean COG-value we calculated the standard deviation only instead of the coefficient of variation. In order to calculate the highest observed variability we again calculated a mean value of the one third of speakers with the highest variability in each of our three groups (flat palates, medium palates, domed palates).

\section{Coefficient of variation of the number of contacts in the row with the most contact - "NOC variability"}

In order to calculate the variability of the number of contacts we used the articulatory target again. From the EPG frame at the target we took the row with the greatest number of contacts (in Figure 3 this would be row 6) and calculated the standard deviation of the numbers of contacts within these rows for the 30 repetitions of an item uttered by a speaker. We divided the standard deviation by the mean (and thereby received the coefficient of variation) since there was a correlation between mean and standard deviation. As for all the other parameters, the highest observed variability was calculated. This variability was called maximally observed NOC-variability.

The aim of calculating this parameter was to investigate the variability of the groove width which is important for the acoustic output. In contrast to COG-variability the NOC- 
variability measures tongue height differences instead of differences in tongue position.

\section{F. Measuring acoustic variability}

Acoustic variability was calculated on the basis of the frequencies of the first three formants. Section II.F.1 describes how the formants were measured. Section II.F.2 describes how the acoustic variability was measured.

\section{Formant measurements}

For the measurement of the first three formants the data were first downsampled from 48 to $10 \mathrm{kHz}$. Afterwards semi-automatic measurements were carried out in Matlab with a signal window length of $20 \mathrm{~ms}$ and a shift duration of $5 \mathrm{~ms}$ (=75\% overlap) between neighbouring windows.

An LPC analysis with 14 coefficients was calculated. Then upper and lower boundaries in the frequency domain were defined for each formant of each speaker and each vowel. The formant detection program then looked for true maxima, corresponding to zero crossing of the first derivative of the spectral envelope. It looked for a unique maximum within each of the three frequency ranges that were defined for each of the first three formants.

For example, for most male speakers, F1 was detected in the [200 $400 \mathrm{~Hz}$ ] range for /i/, while F2 was detected in the $[18002400 \mathrm{~Hz}]$ range for /i/. If there was a frequency range within which no true maximum could be found, the program looked for humps on the envelope. Humps are defined as parts of the envelope, where the first derivative keeps the same sign but varies abruptly around a value close to zero. If at the end of the processing, the LPC analysis did not propose a solution for each of the formant-specific frequency ranges, the whole process was repeated for the cepstral analysis. The results were controlled manually and the boundaries were reset if necessary.

Formant detection is especially complicated when several formants get closer to form a single peak. To give an example, in German, F2 and F3 of the vowel /i/ are usually very 
close so that they could be interpreted as one formant by a formant detector. This is the reason why we decided to use a guided semi-automatic detection, and not an automatic one, and to rely on two methods, an LPC analysis and cepstral analysis, in order to compute the spectral envelope.

In order to better illustrate the perceptual influence of a certain variability, the formants were transformed into barks according to the following formula (Schroeder et al. (1979)):

$$
F_{\text {Bark }}=7 * \operatorname{asinh}\left(F_{H z} / 650\right) .
$$

For some speakers and some items formants could not be reliably measured, or they could be measured for some repetitions only. If more than 10 repetitions could not be reliably measured, the items were excluded from further acoustic analysis since, judging from our corpus, 20 repetitions are needed for obtaining stable measurements for variability (i.e. the variability stops rising if more repetitions are included). If less than 20 repetitions are taken, variability drops.

Consequently, the following data were excluded from further analysis:

- F2 and F3 of /I/ by male speaker E1 where we usually found three peaks instead of two in the F2-F3 region,

- F2 and F3 of /I/ by female speaker N3 (same problem),

- F2 and F3 of /i/ by female speaker N5 where we often had just one peak instead of two in the F2-F3 region,

- F2 of male speaker E9's /I/, where the formant peak was too small in amplitude,

- F2 and F3 of /e/) by female speaker N4, where the formant peaks were too small in amplitude,

- F2 of item /I/ by female speaker N6 where the peak was too small in amplitude. 


\section{Standard deviation of F1, F2, F3 - "F1-variability, F2-variability, F3 variability"}

The calculation of acoustic variability was carried out similarly to the POC variability (section II.E.1), except that no coefficient of variation was measured. As for the articulatory data, a nonlinear time alignment was carried out for the formant values measured over a complete segment. The mean value and the standard deviation of the bark transformed formants were calculated for each of the samples. Then a mean of the standard deviations was calculated for each sound and each speaker. The coefficient of variation was not calculated since there was no correlation between mean and standard deviation.

\section{G. Statistical analyzes}

All statistical analyzes were carried out in SPSS 15.0. The following tests were carried out.

- In order to investigate whether there is a gender effect in palate size we calculated a one-factorial ANOVA with gender as factor and palate shape $(\alpha)$ as dependent variable.

- A possible influence of gender on all the variabilities (POC-variability, COG-variability, NOC-variability, F1-variability, F2-variability, F3-variability) was assessed with a repeated measures ANOVA with item as within-subject factor and gender as betweensubject factor.

- A possible influence of the size of the phoneme inventory on all the variabilities (POC-variability, COG-variability, NOC-variability, F1-variability, F2-variability, F3variability) was assessed with a repeated measures ANOVA with item as within-subject factor and vowel inventory type (2 vs. 3 vs. 4 unrounded front vowels) as betweensubject factor. 
- In order to see whether there is a significant influence of the palate shape on the maximally observed variabilities the palate shapes were divided into three groups (flat, medium and domed palates) and the influence of the palate group on the variabilities was assessed via a repeated measures ANOVA with item as within-subject factor and palate group as between-subject factor.

\section{RESULTS}

This section presents the results for our measurements. At first all the parameters were checked to see if there is a gender effect (section III.A). Then the influence of the phoneme inventory on the parameters was investigated (section III.B). In section III.C the results for the influence of the palate shape on POC-variability, COG-variability and NOC-variability are presented. Then the influence of the palate shape on the acoustic variability is discussed (section III.D). Finally the results of our statistical analysis are presented (section III.E).

\section{A. Gender effect}

Our corpus included data for 15 female and 17 male speakers. After the calculation of our parameter $\alpha$ we tested whether gender has an influence on the palate shape. A onefactorial ANOVA showed that there was none $(\mathrm{F}(31,1)=.721, \mathrm{p}=.403)$. However, as will be seen later in the results section, although our sample seems to be balanced for gender, all the flat palates belong to males.

Furthermore, we tested all our parameters (POC-variability, COG-variability, NOCvariability) to see if there is an influence of gender. The results can be found in table II. As can be seen, there is a significant effect of gender on COG-variability, F1-variability and F2-variability. The influence of gender on F3-variability is close to reaching significance. In all further analyzes of COG-variability, F1-variability, F2-variability and F3-variability we will therefore split data according to gender.

TABLE II 


\section{B. Influence of the size of the phoneme inventory}

In section II.D we noted that Polish and Bulgarian have fewer unrounded front vowels. The results of a repeated measures ANOVA show that the influence of the size of the phoneme inventory does not reach significance (cf. table III).

TABLE III

\section{Relationship between palate shape and articulatory variability}

\section{General findings}

Figure 4 shows as an example the relation between POC-variability and the palate shape found for /I/. On the abscissa the $\alpha$ values of the speakers are displayed and on the ordinate the POC-variability.

\section{FIGURE 4}

As can be seen from the crowdedness of the points in the middle of the figure, the sample is not entirely well balanced with regard to the palate shape. Whereas there are just a couple of speakers with a very curved (low $\alpha$ ) or a very flat palate (high $\alpha$ ), the majority of the speakers clusters around the middle.

Furthermore, as expected, the variability varies more in the left half of the figure (for low $\alpha$ and domed palates) than in the right half (for high $\alpha$ and flat palates). In the left half one can find speakers with both high and low variability. On the right side, however, there are only speakers with low variability. This is consistent with the hypothesis that the speakers with a flat palate all reduce their articulatory variability in order to preserve the acoustic output. For the other speakers, the amount of articulatory variability is more variable: It can be significantly larger than for speakers with a flat palate, but this is not systematically the case.

In general, the expected relation can be seen quite clearly: The maximally observed variability for a certain range of palate shapes decreases from left to right. We will therefore 
go on looking at the results for the maximally observed variability.

Figure 5 shows the percentage of activation of each electrode at the articulatory target for the three groups of palates and each item. Flat palates are shown in the left column, medium palates in the middle and domed palates in the right. Each line shows a different item. White asterisks mean "no contact" over all trials of all speakers with this kind of palate, black asterisks mean "always contact" in all trials of all speakers with this kind of palate. Grey asterisks denote an intermediate activation, dark grey means more than 50\%, light grey less than $50 \%$.

\section{FIGURE 5}

One can see that the most grey asterisks (denoting that this contact is sometimes active, sometimes it is not), and therefore the most variability in contacts, are around the groove in the middle and in the most anterior rows. The variability in groove width is probably due to differences in tongue height: When the tongue is lowered contact in the middle of the palate disappears first. The variability in the front rows is certainly due to differences in tongue position: When the tongue is moved posteriorily contact in the anterior rows disappears.

\section{Percent of contact ("POC-variability")}

Figure 6 shows all the POC-variability values as a function of the palate shape. Each small letter gives the value of one measurement of the variability for one sound and one subject. The vertical lines mark the borders of the three groups of palates needed for the calculation of the maximally observed POC-variability. The domed palates are on the left, the medium palates in the middle and the flat palates on the right. Comparable to figure 4 one can see that the variability varies for speakers with a domed palate but tends to be low for the speakers with a flat palate.

Plotted on top of these values bold letters connected by lines can be seen. These are the mean values of the maximally observed POC-variability in each group. All the lines, except the one for /i/, are falling from left to right. This means that, even if there are speakers with 
a domeshaped palate exhibiting low variability, the maximally observed variability in the group of speakers with domeshaped palates is higher than the maximally observed variability in the group of speakers with a flat palate.

FIGURE 6

\section{3. center of gravity ("COG-variability")}

\section{FIGURE 7}

Figure 7 shows the results for our measurements of COG-variability. Since there was a significant effect of gender, data are split according to gender. As one can see there are no female speakers with flat palates. This contrasts with previous results from the literature where female palates were found to be smaller and thus flatter. This could be due to the relatively small sample of our study as compared to the previous studies (e.g. 48 subjects in Cheng et al. (2007) and 129 in Fitch and Giedd (1999)).

The results do not show the same tendency as for the POC-variability. It rather seems that there is no tendency at all, but that the maximally observed variability is connected to the number of subjects in the group: There are many subjects with a medium palate so there is a high maximally observed COG-variability.

The different results for POC and COG could be due to the fact that the COG measures differences in the front-back dimension whereas the POC measures differences in both dimensions (front-back and laterality).

As can be seen in figure 5, variability occurs both in tongue height and tongue position. So the fact that we do not find results for COG but for POC variability could indicate that only the variability in tongue height is connected to the palate shape.

\section{Number of contacts in the row with most contact ("NOC-variability")}

Figure 8 shows the results of the third method to calculate articulatory variability. The figure shows the coefficient of variation for the contacts in the row with the highest number 
of contacts at the target position.

As one can see, a similar tendency as for the POC-variability can be observed, even if the details vary. For $/ \mathrm{I} /, / \mathrm{j} /$ and $/ \varepsilon /$ one can see that the highest observed variability shown by the big letters and the lines connecting them is highest for the speakers with a domed palate, a little lower for the speakers with a medium palate and lowest for speakers with a flat palate. For the two tense vowels /i/ and /e/ this tendency cannot be observed.

FIGURE 8

\section{Acoustic variability}

According to our rationale in the beginning, speakers with flat palates should have more acoustic variability if they have the same articulatory variability as speakers with a domeshaped palate. Since, however, these speakers have less articulatory variability, they can be expected to have about the same acoustic variability as speakers with domed palates.

Figures 9 to 11 give the results for the variability of the formants. Since a gender effect was found data were split by gender. Same as for COG-variability, it is hard to see a tendency, except for the dependence of the variability on the sample size. Most important, however, the acoustic variability of speakers with flat palates is never greater than that of speakers with domeshaped palates. As discussed in the introduction, there should be higher acoustic variability in speakers with flat palates, if speakers ignored the differences in acoustic variability resulting from different palate shapes.

FIGURE 9

FIGURE 10

FIGURE 11

\section{E. Statistical analysis}

The results of our statistical analysis in table IV show that for the maximally observed POC and NOC-variability there is a significant effect of the form of the palate on the 
articulatory variability. None of the acoustic parameters showed a significant effect; neither did maximally observed COG-variability.

TABLE IV

\section{DISCUSSION}

The aim of the current study was to investigate how morphology influences motor strategies in order to reach an acoustic target.

As has been explained in the introduction, the starting point of our study was the assumption that, without adjustments by the speaker, a given amount of articulatory variability should result in less acoustic variability if the palate is domeshaped than if it is flat, since the constriction area should be modified to a higher degree by articulatory variability in the second case than in the first.

By this reasoning, let us hypothesize that speakers should compensate for the shape of their palate, in order to counteract the impact of the palate shape on the acoustic variability, and to keep this variability within a range compatible with the correct perception of the phoneme. More precisely, speakers with flat palates should reduce their articulatory variability, and there should be a relationship between palate shape and articulatory variability.

In this theoretical framework, articulatory and acoustic variability of 32 speakers of various languages has been measured. In order to assess articulatory variability we calculated the coefficient of variation of the percent of contact (POC-variability), the standard deviation of the center of gravity (COG-variability) and the coefficient of variation of the number of contacts in the row with most contact (NOC-variability). The first one of these measures assesses overall variability, the second variability in the horizontal dimension (tongue position) and the third the variability in the vertical direction (tongue height).

The first main finding of this experimental study is that for 3 of the 5 phonemes that were analyzed, namely /I, $\varepsilon, \mathrm{j} /$, there is clearly a relationship between maximal POC- and 
NOC-variability and palate shape. For a fourth item, /e/, there is one for POC-variability. Indeed, for these sounds, speakers with flat palates show reduced articulatory variability. Large articulatory variability is observed for some speakers with domeshaped palates, while small articulatory variability is systematically observed for speakers with a flat palate. For NOC-variability no consistent results could be found. We therefore conclude that there is a systematic relationship between variability in vertical tongue position but not in horizontal tongue position. This is consistent with our rationale from the beginning which predicted a relation between vertical variability and palate shape.

For one sound, /i/, no trend in either POC- or NOC-variability could be observed. This could be due to the generally low articulatory variability of this sound. Fujimura and Kakita (1979) show that synchronous activity of the different parts of the genioglossus in /i/ lead to a stable articulatory pattern ("stabilization effect") and low acoustic variability ("saturation effect"). Similarly, Perkell (1990, p.269f) described this generally low articulatory variability of high tense vowels as a saturation effect. The tongue blade is stiffened and grooved and pushed against the hard palate by genioglossus posterior activity. In this position the tongue stays rather stable even if activation levels of the genioglossus posterior vary. According to Perkell (1990) this saturation effect would strongly simplify the control of the stability of the tongue positioning for high front vowels, and it would ensure that the acoustic variability remains within a range compatible with a correct perception of the phoneme. This hypothesis is supported by the fact that, in spite of its low articulatory variability, /i/ shows about the same amount of maximal acoustic variability as compared to the other sounds (but see criticisms of the saturation effect hypothesis in Buchaillard et al. (2008)).

The second main finding is that the acoustic variability was experimentally never found to be greater for speakers with flat palates than for speakers with domeshaped palates. This shows that the impact of the palate shape on the amount of articulatory variability counteracts the natural influence of palate flattening in the acoustics, namely the increase in acoustic variability.

Consequently, we interpret the experimental findings of this study as supporting the hy- 
pothesis that, in order to preserve the acoustic correlates of the perception of these phonemes, speakers specifically adapt their articulatory variability to their morphology. More precisely, speakers control the accuracy of their tongue positioning in relation to their palate shape in order to make sure that the acoustic variability remains within a range compatible with the correct perception of the phoneme. For some speakers, i.e. those for whom the amount of acoustic variability is very sensitive to changes in the amount of articulatory variability, the reduction of the articulatory variability even generated a reduction of the acoustic variability which was stronger than necessary (i.e. the acoustic variability was below the one measured for speakers with other palate shapes).

\section{Acknowledgments}

This work was supported by a grant from the German Research Council (PO 334/4-1) and by the POPAART P2R program. We would like to thank Olessia Panzyga and Anke Busler at the Zentrum für Allgemeine Sprachwissenschaft for acoustical labelling and for carrying out palate measurements. We furthermore thank Jim Scobbie, Alan Wrench and Inger Moen for enabling recordings in Edinburgh and Oslo. We are indebted to Jorge C. Lucero who provided a program for the calculation of the temporal alignment. Thanks to Christian Geng and Tine Mooshammer for statistical advice. We are grateful to Laura Koenig, Tine Mooshammer, four anonymous reviewers and the editor Christine Shadle for comments on earlier versions of this paper. Thanks to Jörg Dreyer and Daniel Pape for technical support. Thanks to Marzena Zygis, Dora Tonneva, Hristo Velkov and Inger Moen for help during the development of the speech material. Furthermore, many thanks to the subjects at the Zentrum für Allgemeine Sprachwissenschaft in Berlin, at the QMUC Edinburgh and at the University of Oslo. 


\section{APPENDIX A: COMPUTATION OF THE CROSS-SECTIONAL AREA AND THE COEFFICIENT $\alpha$ IN THE MODEL}

In order to represent the palate and tongue shape we use a double-parabola model. In the following we will describe how the alpha coefficient is derived for this general model and then how it is derived in our special case where we have no information on the tongue surface and therefore assume that the tongue is flat. An advantage of the double-parabola model as compared to the bell-shaped model is that it gives room for further explorations where tongue curvature data might be available.

\section{FIGURE 12}

For the computation of the cross-sectional area in the model the palate and the tongue are represented as (cf. Figure 12):

$$
\begin{aligned}
& y_{\text {pal }}(x)=a_{\text {pal }} x^{2}+y_{0 p} \\
& y_{\text {ton }}(x)=a_{\text {ton }} x^{2}+y_{0 t}
\end{aligned}
$$

for $a_{p a l}<0$, with $y_{0 p}$ being the midsagittal point of the palate and $y_{0 t}$ the midsagittal point of the tongue surface. $a_{t o n}$ could be positive or negative and still be articulatorily meaningful. For our calculations we chose $a_{t o n}=0$. The area below the palate is

$$
\begin{gathered}
A_{p}=\int_{-x_{\max }}^{x_{\max }}\left(a_{p a l} x^{2}+y_{0 p}\right) d x \\
=\frac{1}{3} a_{p a l}\left(x_{\max }^{3}+x_{\max }^{3}\right)+y_{0 p}\left(x_{\max }+x_{\max }\right) \\
=\frac{2}{3} a_{p a l} x_{\max }^{3}+2 y_{0 p} x_{\max }
\end{gathered}
$$

with $x_{\max }$ and $-x_{\max }$ being the x-values of the intersection points of tongue and palate. The area below the tongue is

$$
A_{t}=\frac{2}{3} a_{t o n} x_{\max }^{3}+2 y_{0 t} x_{\max }
$$

The resulting cross-sectional area is the difference between $A_{p}$ and $A_{t}$.

$$
A=\frac{2}{3}\left(a_{\text {pal }}-a_{\text {ton }}\right) x_{\text {max }}^{3}+2\left(y_{0 p}-y_{0 t}\right) x_{\max }
$$


One can now introduce the cross-sectional distance $d_{\text {sagit }}$ as the difference between $y_{0 p}$ and $y_{0 t}$.

$$
A=\frac{2}{3}\left(a_{\text {pal }}-a_{\text {ton }}\right) x_{\text {max }}^{3}+2 d_{\text {sagit }} x_{\text {max }}
$$

Since

$$
y_{\text {pal }}\left(x_{\max }\right)=y_{\text {ton }}\left(x_{\max }\right)
$$

and

$$
\begin{gathered}
x_{\max }>0 \\
a_{\text {pal }}\left(x_{\text {max }}\right)^{2}+y_{0 p}=a_{\text {ton }}\left(x_{\text {max }}\right)^{2}+y_{0 t} \\
x_{\text {max }}=\sqrt{\frac{y_{0 p}-y_{0 t}}{a_{\text {ton }}-a_{\text {pal }}}}
\end{gathered}
$$

for $\left(a_{t o n}-a_{p a l}\right)>0$, otherwise there will be no intersection between tongue and palate. Since the sagittal distance is

$$
\begin{gathered}
d_{\text {sagit }}=y_{0 p}-y_{0 t} \\
x_{\text {max }}=\left(\frac{d_{\text {sagit }}}{a_{\text {ton }}-a_{\text {pal }}}\right)^{\frac{1}{2}}
\end{gathered}
$$

By replacing $x_{\max }$ in formula A8 one gets

$$
\begin{gathered}
A=-\frac{2\left(d_{\text {sagit }}\right)^{\frac{3}{2}}}{3\left(a_{\text {ton }}-a_{\text {pal }}\right)^{\frac{1}{2}}}+\frac{2\left(d_{\text {sagit }}\right)^{\frac{3}{2}}}{\left(a_{\text {ton }}-a_{\text {pal }}\right)^{\frac{1}{2}}} \\
A=\frac{4\left(d_{\text {sagit }}\right)^{\frac{3}{2}}}{3\left(a_{\text {ton }}-a_{\text {pal }}\right)^{\frac{1}{2}}} \\
A=\alpha d_{\text {sagit }}^{1.5}
\end{gathered}
$$

with

$$
\alpha=\frac{4}{3 \sqrt{a_{t o n}-a_{p a l}}}
$$

. If $a_{t o n}=0$

$$
\alpha=\frac{4}{3 \sqrt{0-a_{p a l}}}
$$

which is, since $a_{p a l}<0$, equivalent to Eq. 5 . 


\section{ENDNOTES}

1. Thanks to Phil Hoole for raising this idea.

\section{REFERENCES}

Boersma, P. and Weenink, D. (1992-2004). "Praat, a system for doing phonetics by computer", URL www.praat.org, last viewed: 01/12/2008.

Buchaillard, S., Perrier, P., and Payan, Y. (2008). "Muscle saturation effect in /i/ production: Counterevidence from a 3D biomechanical model of the tongue.", J. Acoust. Soc. Am. 123, 3321.

Cheng, H., Murdoch, B., Goozé, J., and Scott, D. (2007). "Electropalatographic assessment of tongue-to-palate contact patterns and variability in children, adolescents, and adults", Journal of Speech, Language, and Hearing Research 50, 375-392.

Daniloff, R. and Hammarberg, R. (1973). "On defining coarticulation", J. Phon. 1, 239-248. Dixon, R. M. W. (1980). The languages of Australia (Cambridge University Press, Cambridge).

Fant, G. (1966). "A note on vocal tract size factors and non-uniform F-pattern scalings", Speech Transmission Laboratory. Quarterly Progress \& Status Reports 4, 22-30.

Fitch, W. and Giedd, J. (1999). "Morphology and development of the human vocal tract: A study using magnetic resonance imaging", J. Acoust. Soc. Am. 106, 1511-1522.

Fujimura, O. and Kakita, K. (1979). "Remarks on quantitative description of the lingual articulation", in Frontiers of Speech Communication Research, edited by B. Lindblom and S. Ohman, 17-24.

Gimson, A. C. and Cruttenden, A. (2001). Pronunciation of English (Arnold, London). Hardcastle, W. J. and Gibbon, F. (1997). "Electropalatography and its clinical applications", in Instrumental Clinical Phonetics, edited by M. J. Ball and C. Code, 149-193 (Whurr Publishers, London).

Hardcastle, W. J., Gibbon, F. E., and Jones, W. (1991). "Visual display of tongue-palate 
contact: Electropalatography in the assessment and remediation of speech disorders", British Journal of Disorders of Communication 26, 41-74.

Hiki, S. and Itoh, H. (1986). "Influence of palate shape on lingual articulation", Speech Communication 5, 141-158.

Hillenbrand, J., Getty, L. A., Clark, M. J., and Wheeler, K. (1995). "Acoustic characteristics of American English vowels", J. Acoust. Soc. America 97, 3099-3111.

Hoole, P. and Mooshammer, C. (2002). "Articulatory analysis of the German vowel system", in Silbenschnitt und Tonakzente, edited by P. Auer, P. Gilles, and H. Spiekermann, 129-152 (Niemeyer, Tübingen).

IPA (1999). Handbook of the International Phonetic Association: A guide to the use of the International Phonetic Alphabet (Cambridge University Press, Cambridge).

Jassem, W. (2003). "Polish", JIPA 33, 103-107.

Kristoffersen, G. (2000). The Phonology of Norwegian (Oxford University Press, Oxford). Lucero, J. C. and Koenig, L. L. (2000). "Time normalization of voice signals using functional data analysis", J. Acoust. Soc. Am. 108, 1408-1420.

Lucero, J. C., Munhall, K. G., Gracco, V. L., and Ramsay, J. O. (1997). "On the registration of time and the patterning of speech movements", J. Speech Lang. Hear. Res. 40, 1111-1117. Mooshammer, C., Perrier, P., Fuchs, S., Geng, C., and Pape, D. (2004). "An EMMA and EPG study on token-to-token variability", AIPUK 36, 47-63.

Perkell, J. (1990). "Testing theories of speech production: Implications of some detailed analyses of variable articulatory data", in Speech production and speech modelling, edited by W. J. Hardcastle and A. Marchal, 263-288 (Kluwer Academic Publishers, Dordrecht, The Netherlands).

Perkell, J. S. (1997). "Articulatory processes", in The Handbook of Phonetic Sciences, edited by W. J. Hardcastle and J. Laver, 333-370 (Blackwell, Oxford and Cambridge, Massachusetts).

Perrier, P., Boë, L. J., and Sock, R. (1992). "Vocal tract area function estimation from midsagittal dimensions with CT scans and a vocal tract cast: Modelling the transition with 
two sets of coefficients", J. Speech Hearing Res. 35, 53-67.

Peterson, G. and Barney, H. (1952). "Control methods used in the study of vowels", J. Acoust. Soc. America 24, 175-184.

Schroeder, M., Atal, B., and Hall, J. (1979). "Objective measure of certain speech signal degradations based on masking properties of human auditory perception", in Frontiers of Speech Communication, edited by B. Lindblom and S. Ohman (Academic Press, London). Stevens, K. N. (1972). "The quantal nature of speech: evidence from articulatory-acoustic data", in Human Communication: A Unified View, edited by E. E. Davis and P. B. Denes, 51-66 (McGraw-Hill, New York).

Tabain, M. and Butcher, A. (1999). "Stop consonants in Yanyuwa and Yindjibarndi: A locus equation perspective", J. Phon. 27, 333-357.

Vorperian, H., Kent, R., Lindstrom, M., Kalina, C., Gentry, L., and Yandell, B. (2005). "Development of vocal tract length during early childhood: A magnetic resonance imaging study", J. Acoust. Soc. Am. 177, 338-350.

Wrench, A. A. (2007). "Advances in EPG palate design", International Journal of SpeechLanguage Pathology 9, 3-12. 
TABLE I. Number of phonemic contrasts. Leftmost column gives sound class.

\begin{tabular}{cccccc}
\hline \hline & Bulgarian & Polish & English & Norwegian & German \\
\hline palatal or velar approximants & 1 & 1 & 1 & 1 & 1 \\
unrounded front vowels & 2 & 3 & 4 & 4 & 4 \\
\hline \hline
\end{tabular}


TABLE II. Results of repeated measures ANOVA with item as within-subject factor and gender as between-subject factor.

\begin{tabular}{llc}
\hline \hline Parameter & Effect & $\mathrm{F}(\mathrm{p})$ \\
\hline POC-variability & gender & $\mathrm{F}(1,30)=2.68, \mathrm{p}=.112$ \\
COG-variability & gender & $\mathrm{F}(1,30)=4.820, \mathbf{p}=\mathbf{. 0 3 6}$ \\
NOC-variability & gender & $\mathrm{F}(1,30)=.037, \mathrm{p}=.849$ \\
\hline F1 variability, & gender & $\mathrm{F}(1,30)=57.326, \mathbf{p}=\mathbf{. 0 0 0}$ \\
F2 variability & gender & $\mathrm{F}(1,30)=29.688, \mathbf{p}=\mathbf{. 0 0 0}$ \\
F3 variability & gender & $\mathrm{F}(1,30)=2.959, \mathrm{p}=.096$ \\
\hline \hline
\end{tabular}


TABLE III. Results of repeated measures ANOVA with item as within-subject factor and vowel inventory type (number of unrounded front vowels) as between-subject factor.

\begin{tabular}{lcc}
\hline \hline Parameter & Effect & $\mathrm{F}(\mathrm{p})$ \\
\hline POC-variability & vowel inventory type & $\mathrm{F}(2,29)=1.460, \mathrm{p}=.249$ \\
COG-variability & vowel inventory type & $\mathrm{F}(2,29)=0.141, \mathrm{p}=.869$ \\
NOC-variability & vowel inventory type & $\mathrm{F}(2,29)=.138, \mathrm{p}=.871$ \\
\hline F1 variability, & vowel inventory type & $\mathrm{F}(2,29)=2.012, \mathrm{p}=.152$ \\
F2 variability & vowel inventory type & $\mathrm{F}(2,29)=.027, \mathrm{p}=.973$ \\
F3 variability & vowel inventory type & $\mathrm{F}(2,29)=1.594, \mathrm{p}=.220$ \\
\hline \hline
\end{tabular}


TABLE IV. Results of repeated measures ANOVA with item as within-subject factor and palate group as between-subject factor.

\begin{tabular}{clc}
\hline \hline Parameter & Effect & $\mathrm{F}(\mathrm{p})$ \\
\hline POC-variability & palate group & $\mathrm{F}(2,9)=5.698, \mathrm{p}=. \mathbf{0 2 5}$ \\
COG-variability, females & palate group & $\mathrm{F}(1,4)=.014, \mathrm{p}=.293$ \\
COG-variability, males & palate group & $\mathrm{F}(2,3)=3.842, \mathrm{p}=.149$ \\
NOC-variability & palate group & $\mathrm{F}(2,9)=4.262, \mathrm{p}=.05$ \\
\hline F1 variability, females & palate group & $\mathrm{F}(1,4)=1.813, \mathrm{p}=.249$ \\
F1 variability, males & palate group & $\mathrm{F}(2,3)=1.747, \mathrm{p}=.314$ \\
\hline F2 variability, females & palate group & $\mathrm{F}(1,4)=.015, \mathrm{p}=.909$ \\
F2 variability, males & palate group & $\mathrm{F}(2,3)=.177, \mathrm{p}=.846$ \\
\hline $\mathrm{F} 3$ variability, females & palate group & $\mathrm{F}(1,4)=.926, \mathrm{p}=.390$ \\
$\mathrm{~F} 2$ variability, males & palate group & $\mathrm{F}(2,3)=.588, \mathrm{p}=.609$ \\
\hline \hline
\end{tabular}




\section{LIST OF FIGURES}

FIG. 1 Schematisation of the influence of articulatory variability on the crosssectional area for speakers with flat palates (left side) and domeshaped palates (right side). Given the same distance $a$ between the molars (squares), the same variation $\Delta d$ of the sagittal distance, and the same cross-sectional area $A$ for both palates, $\Delta A$ (the wide quadrilateral) is greater for the flat palate than for the domeshaped palate. $d_{f}$ : cross-sectional distance for flat palate, $d_{c}$ : cross-sectional distance for domeshaped palate, $h_{f}$ : height of the quadrilateral (see text), $h_{c}$ : height of the domeshaped palate, $a$ : width of the vocal tract at the height of the molars, $a_{t}$ : width of the vocal tract at the height of the tongue surface for the domed palate. For the flat palate $a_{t}$ would be equal to $a$ and is therefore not shown. . . . . . . . . . . . . .

FIG. 2 Coordinates of the palate electrodes (two points were added laterally in the front row). The curvature of the palate was estimated for the sixth row. Distances are in mm. $\alpha$ for this palate is 1.8062 (medium palate) . . . . . .

FIG. 3 Left: EPG frames for /i/ surrounded by /t/. Time goes from left to right and from top to bottom. White circles represent electrodes without contact, filled circles electrodes with contact. Right: Percent of contact for the EPG frames of /i/ shown on the left (with linear interpolation). Data from speaker

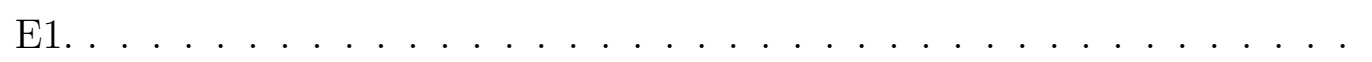

FIG. 4 Relationship between articulatory variability (ordinate) and $\alpha$ (abscissa) for

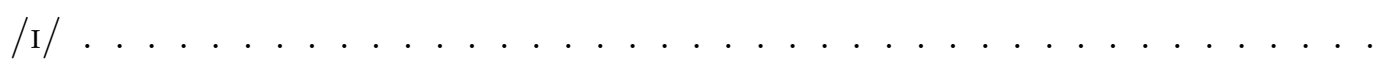

FIG. 5 Mean contact patterns for the five items (rows) and palate types (columns). Black asterisks denote $100 \%$ contact (contact over all repetitions of all speakers in that palate group), white asterisks denote $0 \%$ contact. . . . . . . . . 
FIG. 6 Relationship between $\alpha$ (abscissa) and POC-variability (ordinate). Small letters show results of single measurements. The lines connecting big letters show maximally observed POC-variability. Vertical lines show borders between palate groups. . . . . . . . . . . . . . . .

FIG. 7 Relationship between $\alpha$ (abscissa) and the standard deviation of the center of gravity (ordinate). Small letters show results of single measurements. The lines connecting big letters show maximally observed COG-variability. Vertical lines show borders between palate groups. . . . . . . . . . . .

FIG. 8 Relationship between $\alpha$ (abscissa) and the coefficient of variation of the contact observed in the row with most contact at the articulatoy target (NOCvariability). Small letters show results of single measurements. The lines connecting big letters show maximally observed NOC-variability. Vertical lines show borders between palate groups. . . . . . . . . . . . . . 46

FIG. 9 As figure 6, but for F1-variability and maximally observed F1-variability. . . 47

FIG. 10 As figure 6, but for F2-variability and maximally observed F2-variability. . . 48

FIG. 11 As figure 6, but for F3-variability and maximally observed F3-variability. . . 49

FIG. 12 Calculation of the cross sagittal area and the coefficient $\alpha$. The tongue and the palate are represented by biparabolic equations. The cross-sectional area is calculated as the difference between the area under the palate and the area under the tongue. $\alpha$ can then be calculated from the area and the cross-sectional distance as $\alpha=\frac{4}{3 \sqrt{a_{t o n}-a_{\text {pal }}}}($ Perrier et al., 1992) . . . . . . . 50 


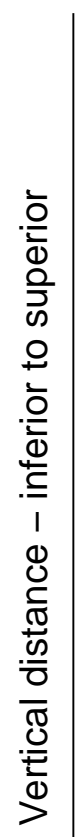

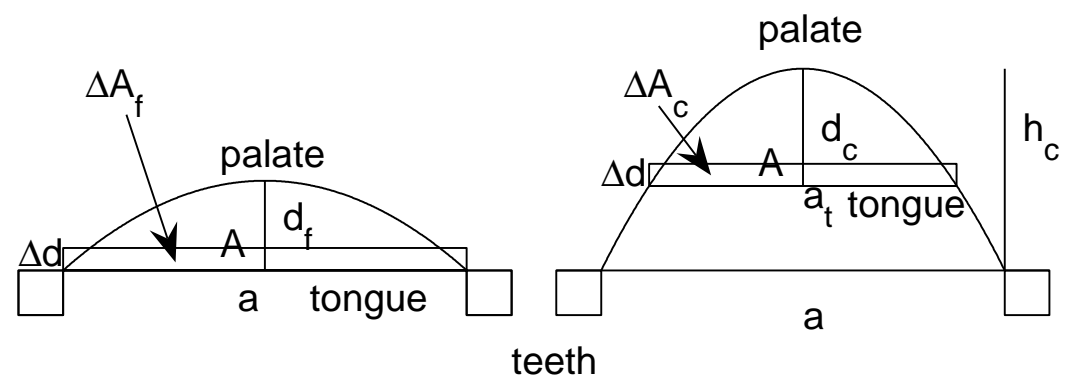

Lateral distance - left to right

FIG. 1. 


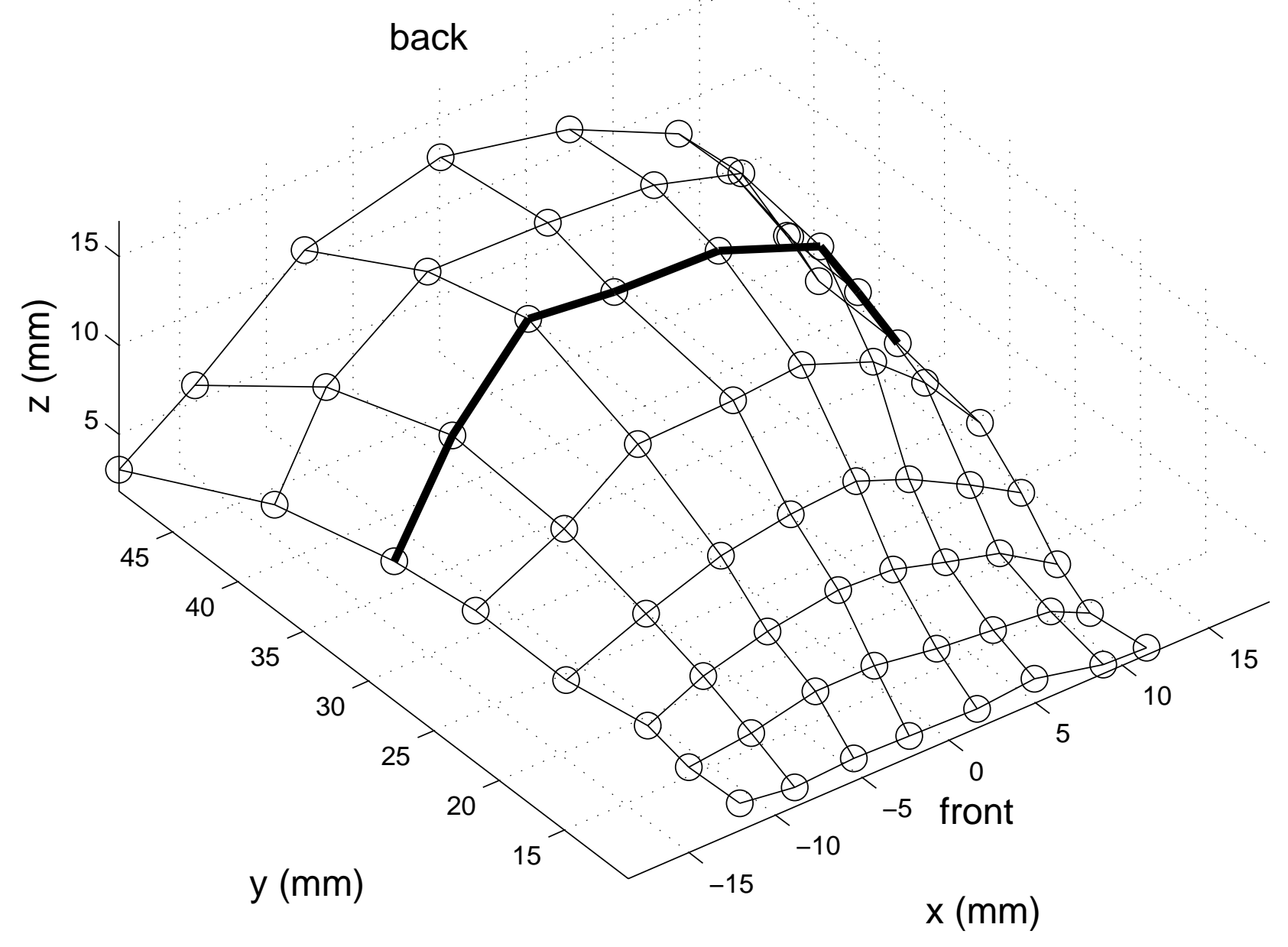

FIG. 2. 


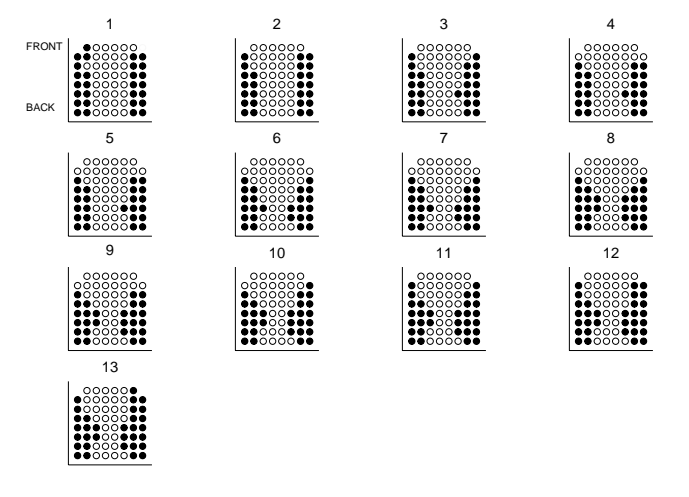

(a)EPG frames

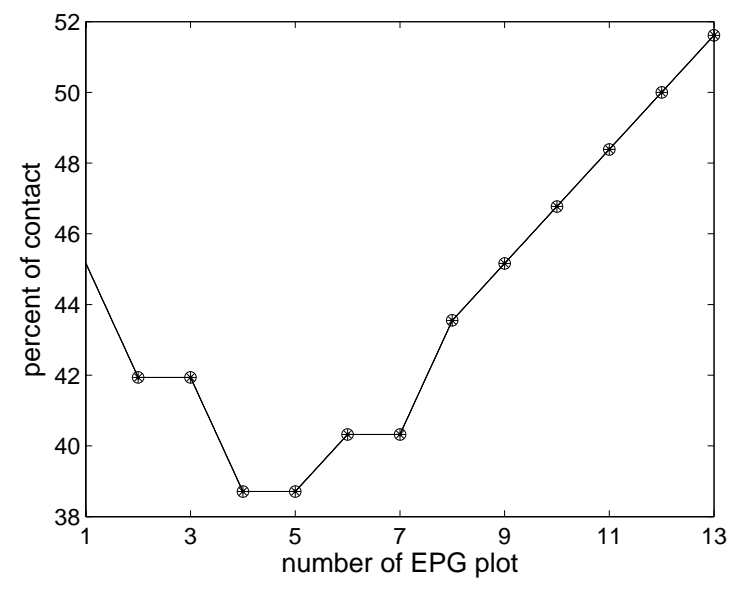

(b)Percent of contact

FIG. 3. 


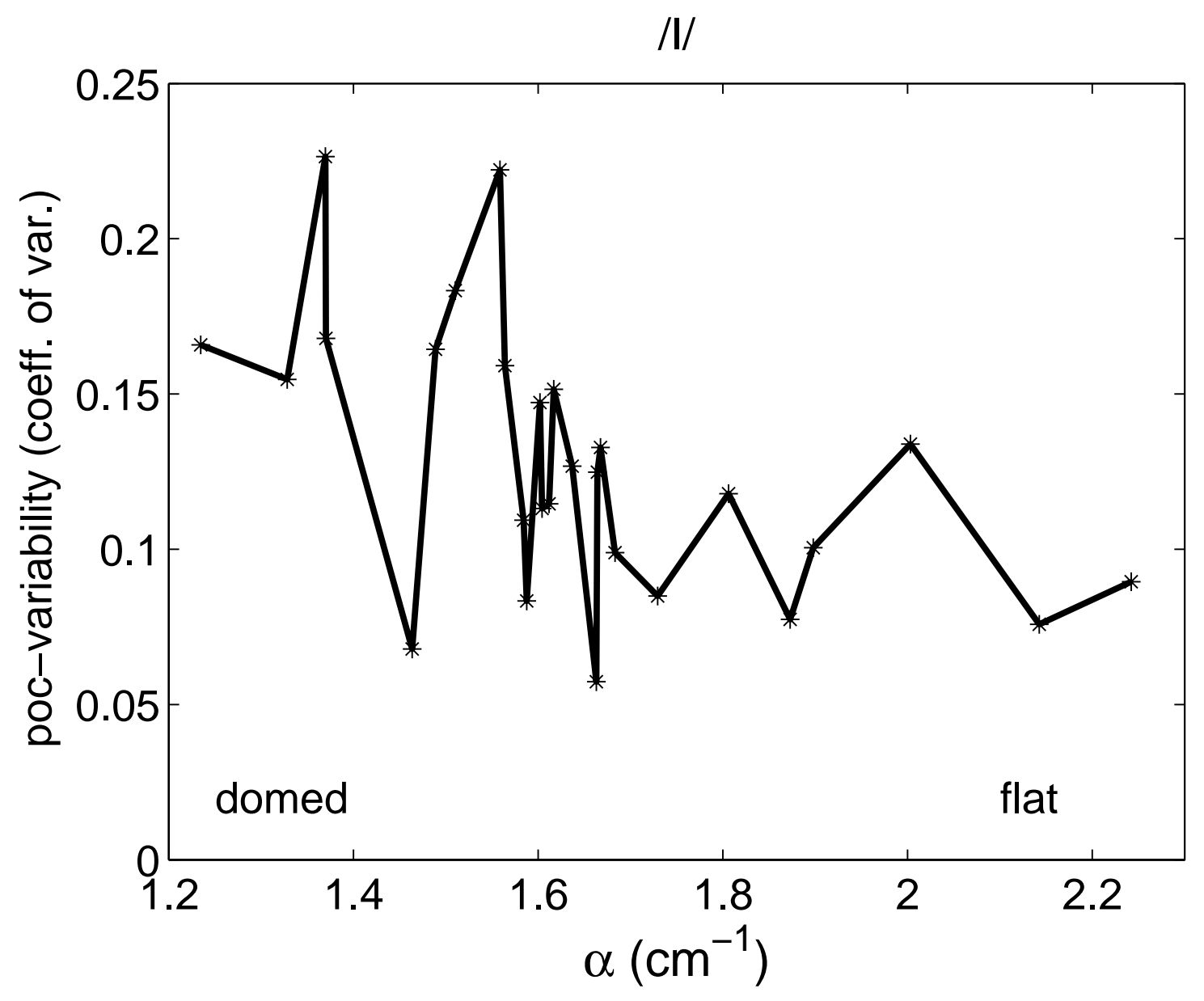

FIG. 4. 


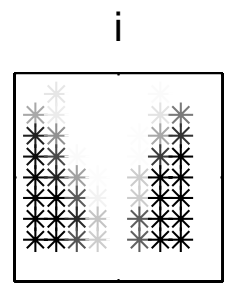

flat

I

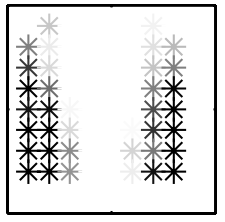

flat

e

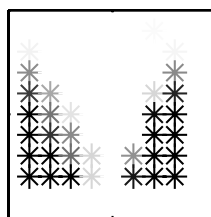

flat

E

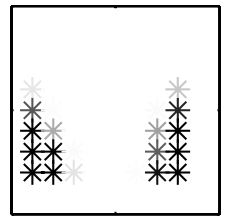

flat

j

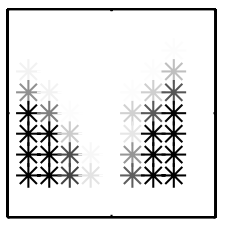

flat

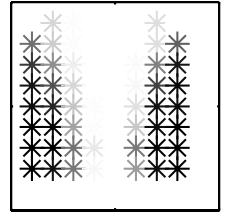

medium

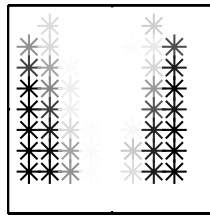

medium

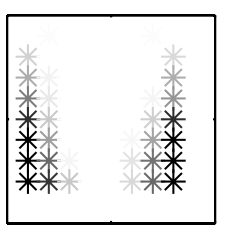

medium

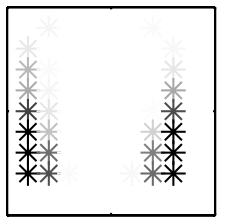

medium

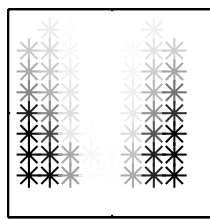

medium

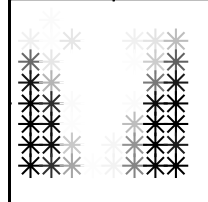

domed

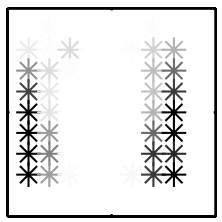

domed

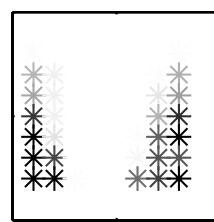

domed
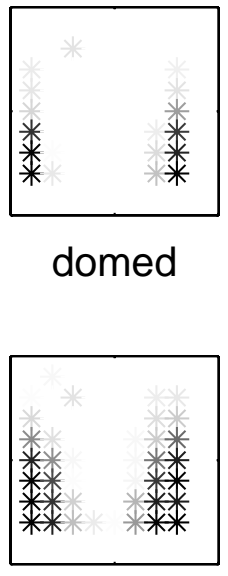

domed

FIG. 5. 


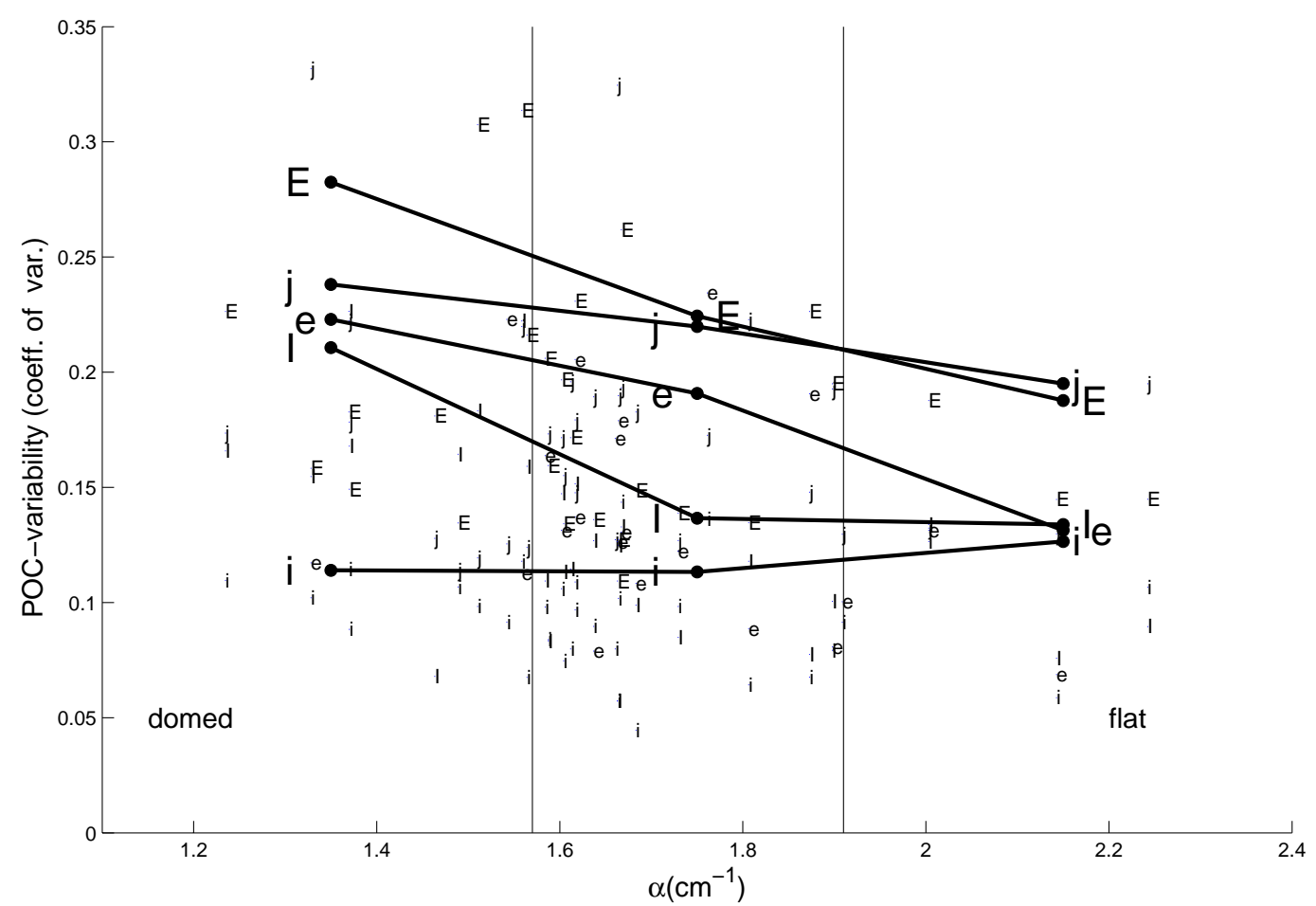

FIG. 6. 

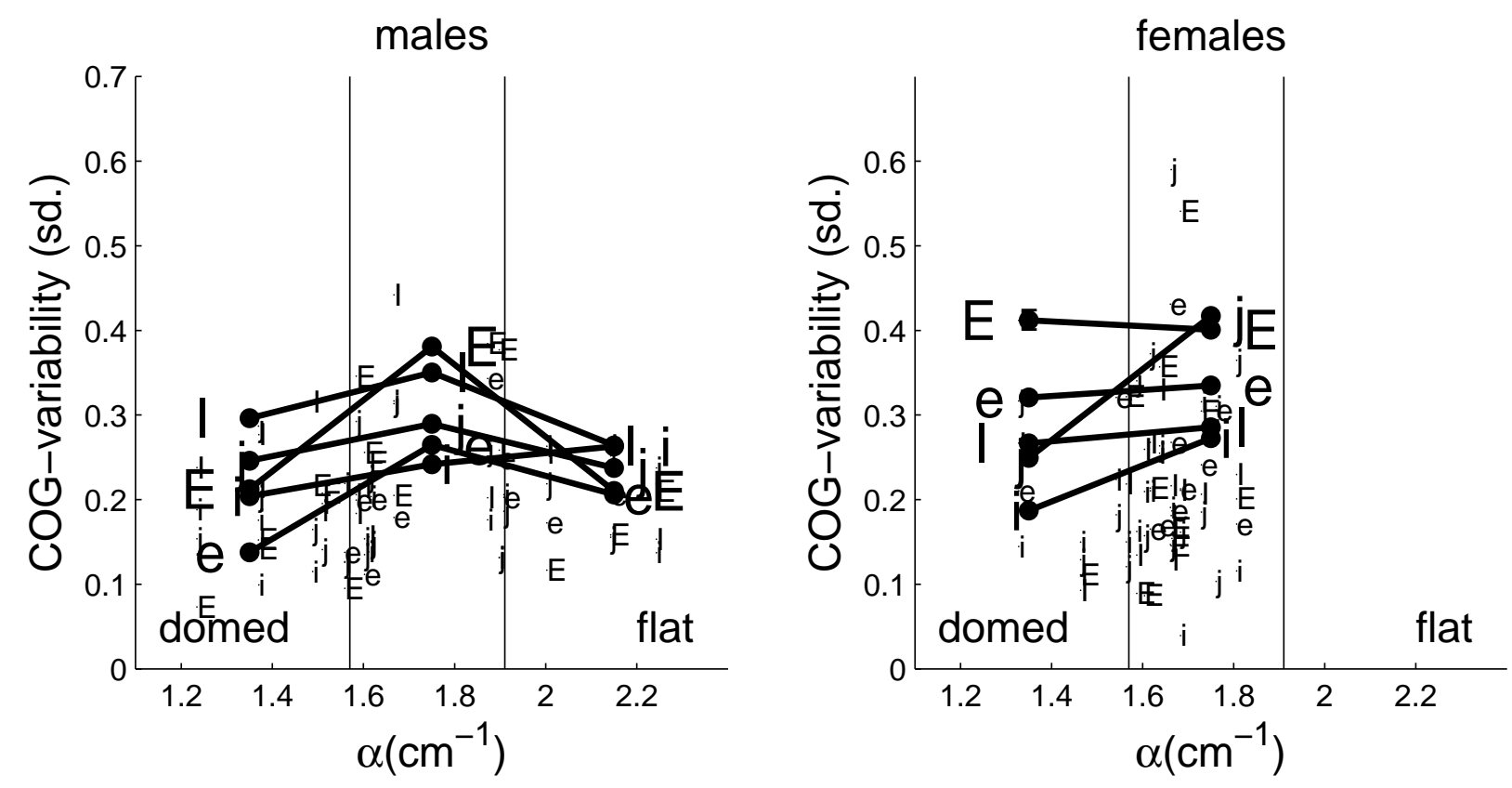

FIG. 7. 


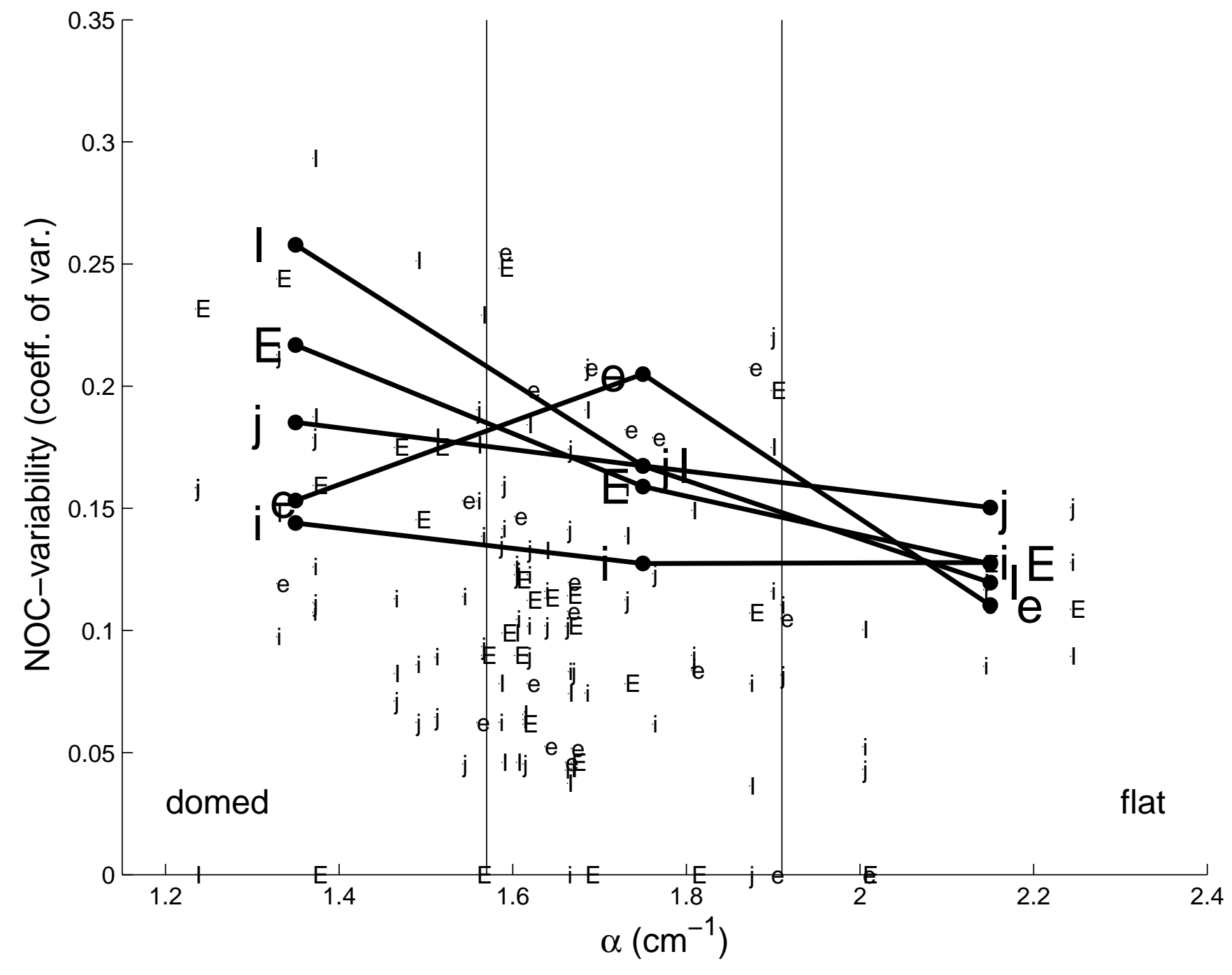

FIG. 8. 

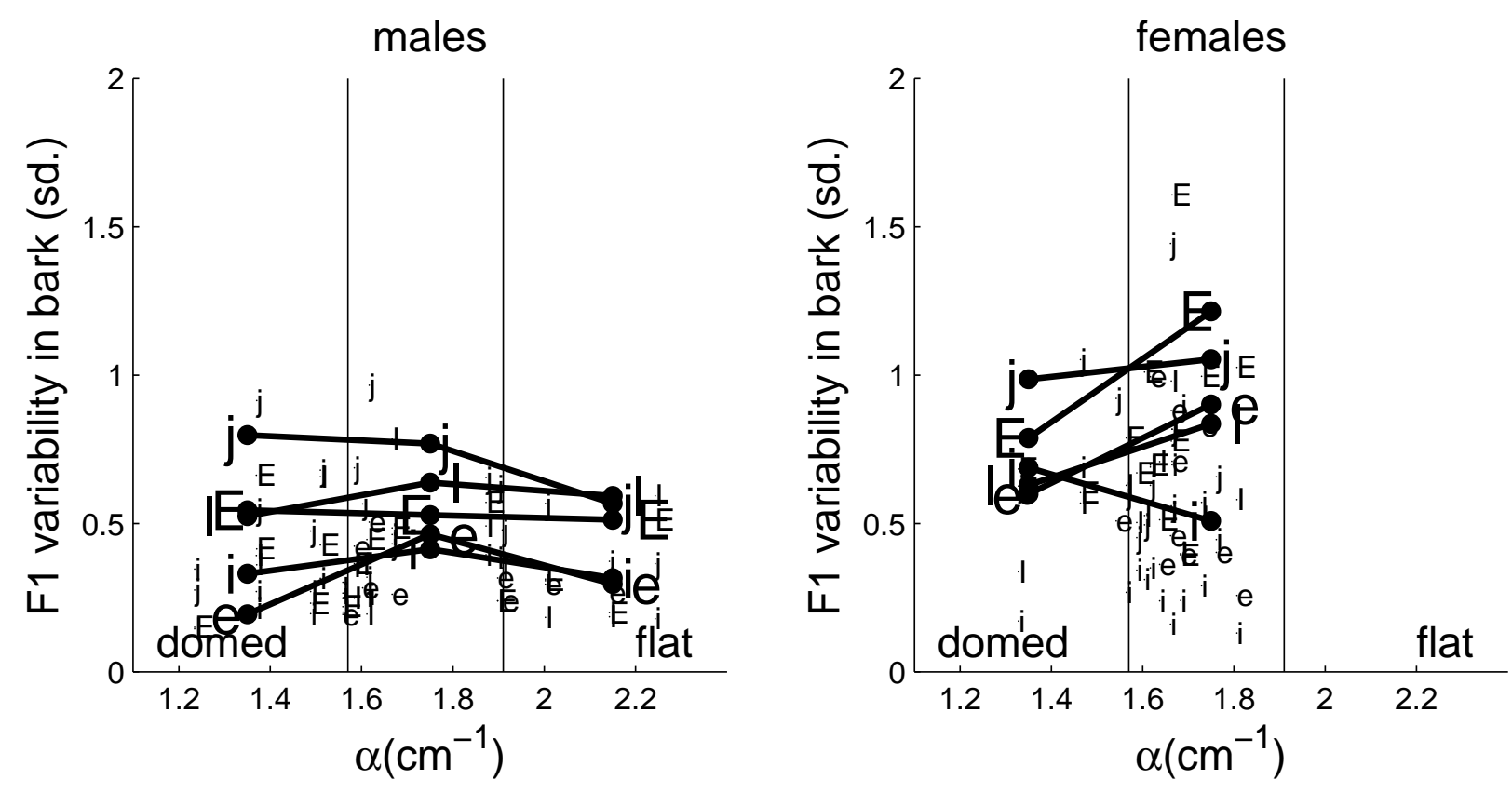

FIG. 9. 

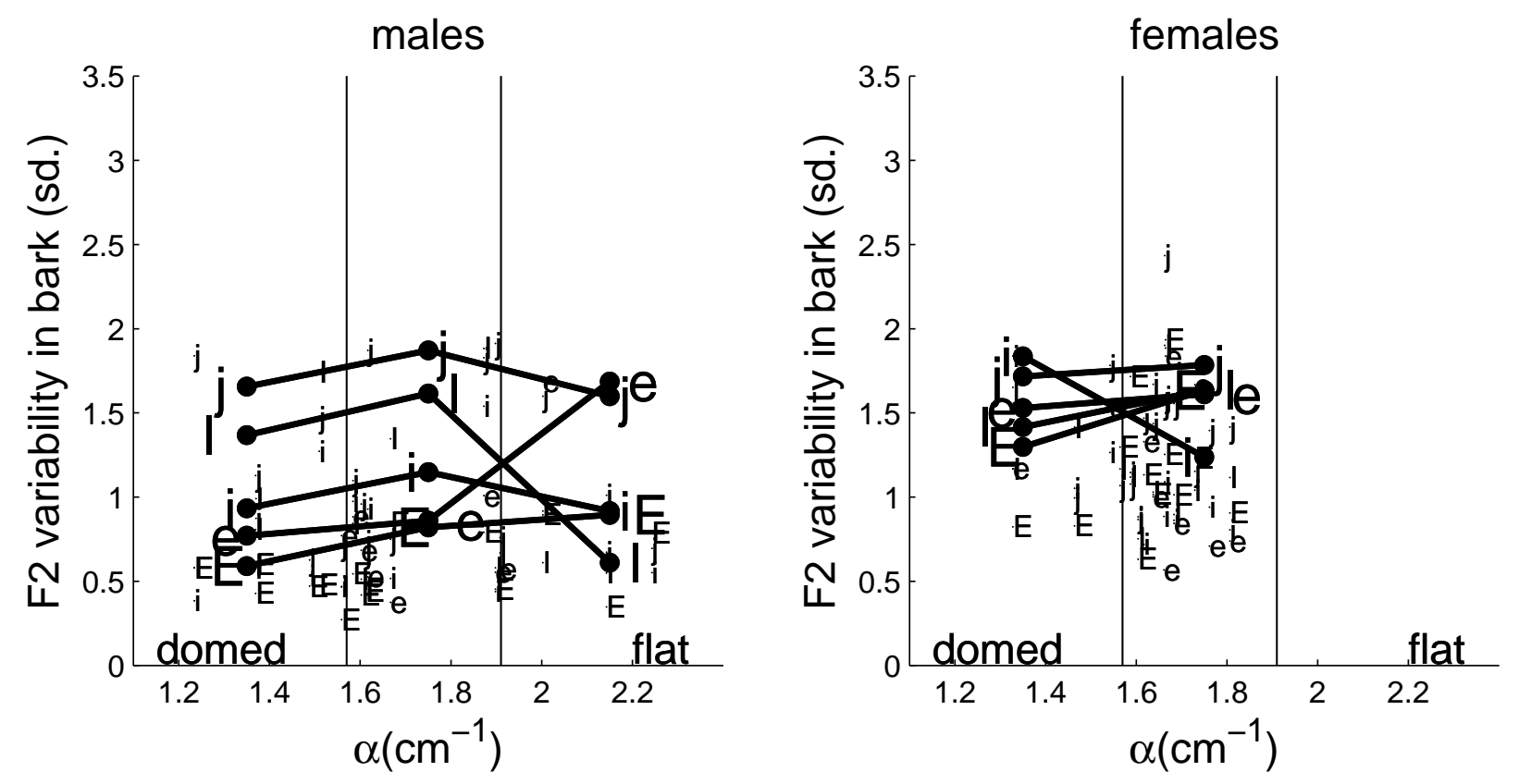

FIG. 10. 

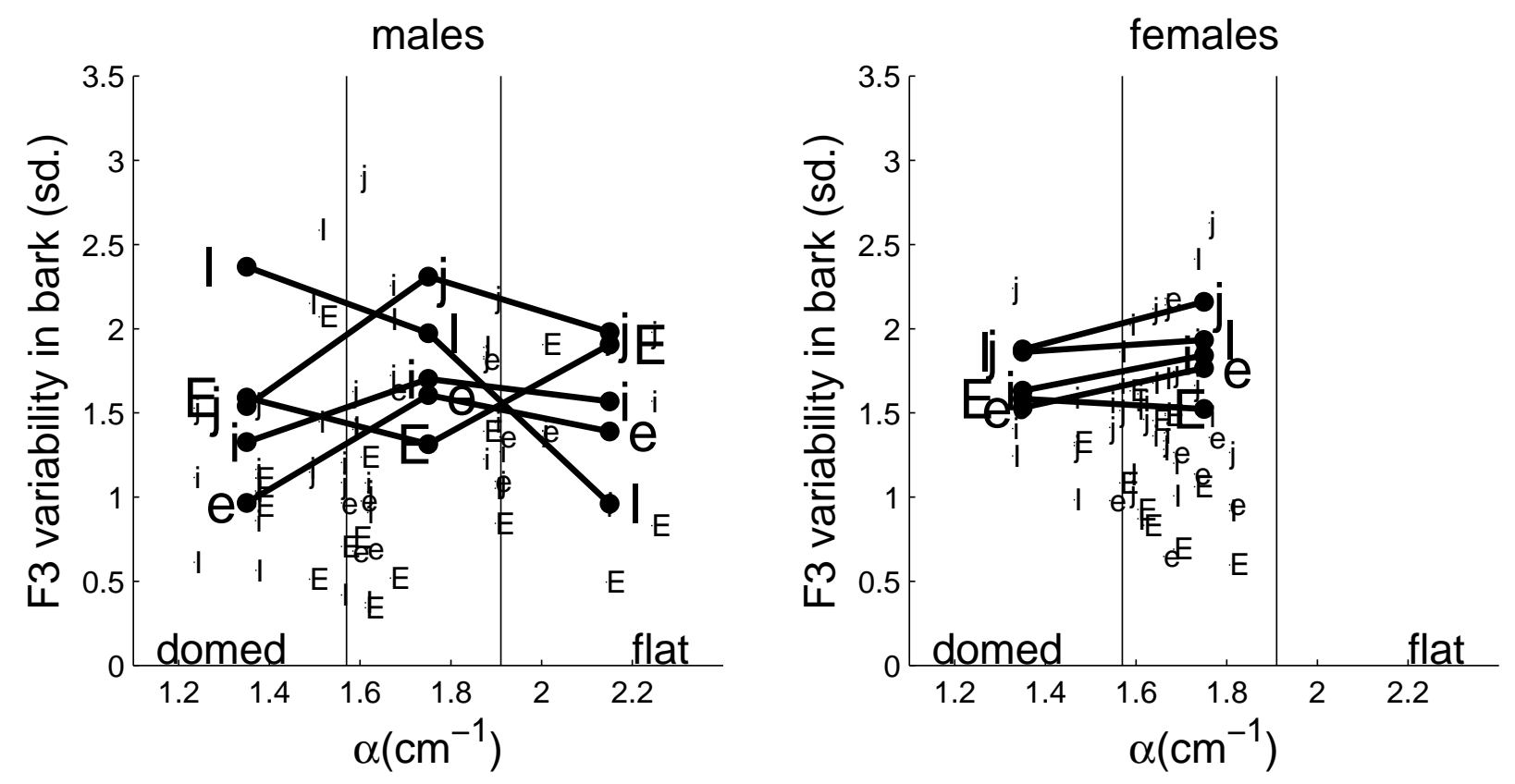

FIG. 11. 


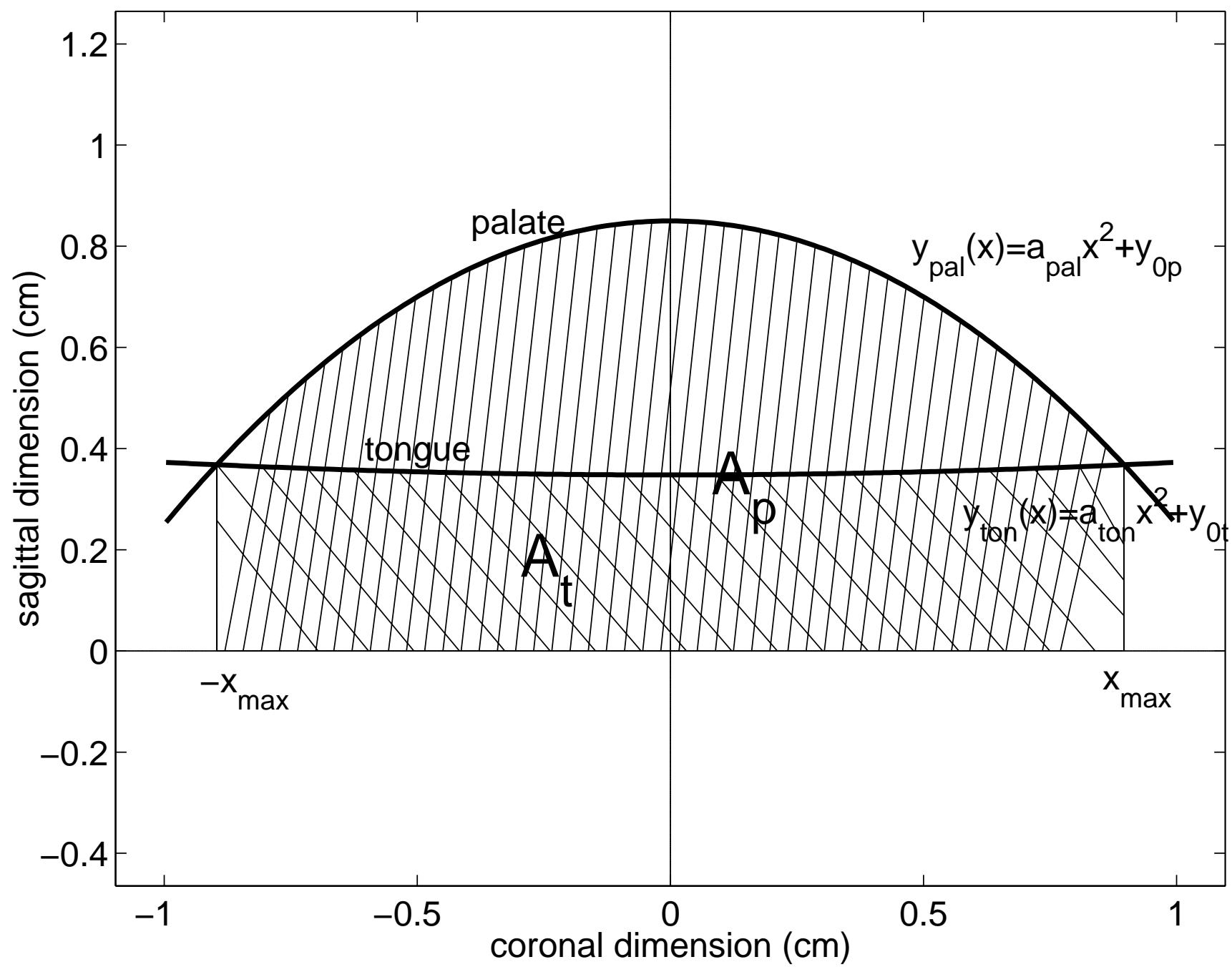

FIG. 12. 